\title{
CONSULTA A ESPECIALISTAS COMO PROCEDIMENTO DE AVALIAÇÃO DE ORIENTAÇÕES PARA O DESIGN DE PUBLICAÇÕES DIGITAIS SISTEMÁTICAS
}

\author{
Maurício Elias Dick \\ Universidade Federal de Santa Catarina \\ mauricioedick@gmail.com \\ Berenice Santos Gonçalves \\ Universidade Federal de Santa Catarina \\ berenice@cce.ufsc.br
}

\begin{abstract}
Resumo: $O$ estudo apresentado neste artigo busca contribuir para que as publicações digitais sistemáticas tornem-se produtos maduros, que apresentem uma linguagem própria - levando em conta as particularidades da mídia e oportunizando a exploração das capacidades do ambiente digital, sem desconsiderar o seu caráter editorial. Assim, a partir do contexto mais amplo de uma pesquisa que buscava construir orientações para o design de publicações digitais sistemáticas, este artigo apresenta o procedimento metodológico de consulta a especialistas que teve por objetivo avaliar as orientações geradas a partir da revisão de literatura. Para a realização do procedimento, foi elaborado um questionário, realizado um teste piloto e posteriormente efetuadas as consultas finais, cujos resultados foram compilados e são apresentados neste artigo. Ao se avaliar o procedimento, entende-se que sua realização trouxe contribuições que não haviam sido identificadas na revisão de literatura, como por exemplo, as dimensões de avaliação e testes da publicação durante o seu desenvolvimento, além de aspectos vinculados à distribuição. Desse modo, a consulta resultou em modificações importantes em relação à primeira configuração do conjunto de orientações, tais como a caracterização do eixo de Superfície, além da readequação dos eixos de Funcionalidades e de Conteúdo.
\end{abstract}

Palavras-chave: design editorial, design digital, publicações digitais sistemáticas, consulta a especialistas.

Abstract: The study presented in this article seeks to contribute to the systematic digital publications to become mature products, that have their own language - taking into account the particularities of the media and providing opportunities to exploit the capabilities of the digital environment, without disregarding their editorial character. Thus, from the broader context of a study that sought to build orientations for the design of systematic digital publications, this article presents the methodological procedure of expert consultation that aimed to evaluate the orientations 
generated from the literature review. To perform the procedure, a survey was designed, a pilot test was conducted and then the final consultation was made, whose results were compiled and are presented in this article. When evaluating the procedure, it is understood that its realization brought contributions that had not been identified in the literature review, for example, the publication's dimensions of evaluation and testing during its development and aspects related to distribution. Thus, the consultation resulted in significant changes in relation to the first set of orientations, such as the characterization of Surface axis, as well as the readjustment of the Features and the Content axes.

Keywords: editorial design, digital design, systematic digital publications, expert consultation.

\section{INTRODUÇÃO}

O design de publicações digitais, tanto sistemáticas, representadas por livros, brochuras e catálogos, quanto periódicas - tais como jornais e revistas - requer do designer um apanhado de definições de projeto que envolvem tanto questões ligadas ao design editorial quanto aspectos do design digital (DICK, 2015). Tal necessidade se dá frente ao seu caráter híbrido, como a própria nomenclatura sugere.

Diante disso, para evoluírem e tornarem-se produtos maduros que apresentem uma linguagem própria, as publicações digitais devem avançar para além da simples mimetização de seus equivalentes impressos, levando em conta também os preceitos do design digital. É necessário, portanto, um conjunto de orientações próprio que considere as particularidades desta mídia e que oportunize a exploração das capacidades do ambiente digital, sem desconsiderar o seu caráter editorial.

Frente a essa necessidade, em um contexto mais amplo de pesquisa, buscou-se construir orientações para o design de publicações digitais sistemáticas (DICK, 2015). Assim, a construção de tais orientações se deu inicialmente a partir da organização, sistematização e interpretação dos fundamentos advindos de uma revisão bibliográfica e outra sistemática.

Já a segunda etapa metodológica envolveu uma consulta a especialistas da área, de modo a avaliar o conjunto desenvolvido, além de oportunizar eventuais contribuições sobre aspectos que não houvessem sido contemplados a partir da revisão de literatura. Assim, este artigo apresenta o procedimento de consulta a especialistas e tem por objetivo mostrar a relevância desta para a construção final do conjunto de orientações. Para a realização do procedimento, foi elaborado um questionário, realizado um teste piloto e posteriormente efetuadas as consultas finais, cujos resultados foram compilados e são apresentados neste artigo.

\section{PUBLICAÇÕES DIGITAIS}

Recentemente impulsionada pela disseminação dos computadores de mão e tablets, as publicações digitais surgiram como novas formas de fazer uso dos recursos e potencialidades oferecidos pelo ambiente digital, explorando o dinamismo e a interatividade entre conteúdo e leitor, agora também usuário. Desse modo, novas possibilidades ao campo do Design surgiram, implicando não somente em uma nova 
alfabetização do designer, mas também em uma mudança de paradigma organizacional, como afirmam Silva e Borges (2011).

A partir de Samara (2011), entende-se que a publicação é um objeto informacional complexo, resultado da aplicação de abordagens conceituais relativas à organização da informação, leiaute e legibilidade. À luz do contexto editorial impresso, o autor afirma que publicações são aplicações extensas de texto e imagem que constituem uma forma unificada e constroem uma comunicação que é maior que a soma de suas partes. Para o autor, as publicações que são "seriadas e evolutivas, ocorrendo periodicamente com novos componentes que se acumulam sobre a ocorrência anterior" (SAMARA, 2011, p.13, grifo dos autores) são classificadas como periódicas. Como exemplo, tem-se as revistas, os jornais e os boletins.

Já as publicações que ocorrem agrupadas ou em diversas ocasiões como partes individuais de um grupo maior são nomeadas como sistemáticas, podendo ser seriadas ou não (no caso de uma coleção fechada, com início e fim predeterminados). Para este propósito, tem-se como exemplos, livros, relatórios e famílias de brochuras.

Em vias de um entendimento comparativo, Craig Mod (2012) afirma que, enquanto no material impresso tem-se a página como a área de composição, nos meios digitais a tela é apenas a fronteira física do dispositivo, sendo a área da publicação, portanto, estendida para além de suas bordas de maneira ilimitada. A partir dessa noção de espaço expandido, para o autor, tem-se a oportunidade de redefinir os modos de conversação entre leitor e conteúdo. Nessa direção, Mod (2012) classifica o livro digital por meio do seu conteúdo, em uma abordagem que independe de suporte, classificação que é compreendida como aplicável a outras publicações do contexto digital. Para ele, então, o conteúdo pode ter sua forma indefinida (conteúdo amorfo ou formless content) ou definida (conteúdo definido ou definite content).

O conteúdo amorfo é independente, podendo ser refluído em diferentes formatos sem perder significado (MOD, 2012), sendo composto principalmente por texto, dada a complexidade de programação e exibição. Já o conteúdo definido se utiliza da área do suporte para arranjar seus elementos (sem limitar-se a ela), não estando impedido de ser refluído, porém sujeito a mudanças de significado decorridos dessa alteração. Este tipo de conteúdo, conforme o autor, geralmente é formado de elementos multimídia, onde se entende mídia como canal de linguagem. De modo similar, a Universidade de Aalto, na Finlândia (AALTO, 2014), também propõe uma categorização das publicações digitais tendo em vista a forma. Assim, considera publicações de forma não distinta e publicações com forma visual distinta.

Como exemplos de publicações digitais de forma não distinta tem-se os livros digitais de leiaute fluído - em formato ePub - cuja configuração visual do conteúdo se adapta conforme o equipamento ou as preferências do leitor. Já as publicações digitais com forma visual distinta são exemplificadas por diversas revistas, jornais e catálogos de leiaute fixo disponíveis para tablets, assim como os chamados livros aplicativos (book apps), geralmente interativos e multimídia.

\subsection{Design de publicações digitais sistemáticas: um conjunto de orientações}

Os princípios e fundamentos do Design Editorial, ainda que essenciais ao design de publicações digitais, não são capazes de suprir as especificidades dessas novas mídias, pois, não contemplam os aspectos interfaciais e da interação humanocomputador que se dão na comunicação e na utilização do ciberespaço. 
Assim, é preciso buscar conceitos chave no campo do Design de Interação que podem ser referências, pois as publicações digitais são interfaces interativas que assumem qualidades e potencialidades específicas oriundas do ambiente digital. Princípios relacionados ao design de interfaces e ao design centrado no ser humano se mostram essenciais para a concepção de publicações digitais que sejam devidamente adequadas aos seus usuários nos diferentes eixos que as configuram.

Desse modo, ao se identificar os princípios existentes nas áreas componentes, busca-se a criação de alicerces próprios para a concepção de projetos específicos, configurando então uma contribuição para a maturidade da mídia.

As contribuições de Samara (2011) trazem um entendimento claro dos principais aspectos que envolvem estes tipos de projetos: a organização do conteúdo, a tipografia, a estrutura e a forma. Junto a essa abordagem, o conceito de grid trazido por Haslam (2007) também reforça a importância da estrutura adequada à publicação, pois esta auxilia na disposição do conteúdo, influenciando na forma e no ritmo final.

No que tange o Design Digital, os referenciais trazidos por Rogers, Preece e Sharp (2013) - somados aos princípios gerais de Lidwell, Holden e Butler (2010) e Schlatter e Levinson (2013) - proporcionam a compreensão dos princípios básicos que envolvem o design de interfaces - em especial a consistência, a visibilidade e a hierarquia, juntamente do conceito de usabilidade visual. Nessa direção, a compreensão do conceito de affordance - conforme Gibson (1986), Cooper (2007) e Gaver (2002) - também contribui para a concepção de interfaces que sejam claras, coesas e de fácil utilização, pois estas se encontram em um ambiente cuja tangibilidade se apoia principalmente no uso de recursos visuais, estando fortemente ligadas à consistência de aplicação (DICK; GONÇALVES, 2014).

Ainda, a pesquisa de Heikkilä (2013) - com enfoque na usabilidade juntamente com os estudos de Compton (2014) e Scherdien (2014) contribuem em grande medida para o entendimento das potencialidades das publicações no ambiente digital, além da compreensão das características destes artefatos.

Partindo-se destes e outros estudos levantados em uma revisão de literatura realizada a partir de uma pesquisa bibliográfica e uma revisão sistemática ${ }^{1}$ em um contexto mais amplo de pesquisa, executou-se a categorização dos principais fundamentos encontrados, buscando auxiliar na compreensão dos diferentes tipos de contribuições dos achados teóricos (DICK, 2015). Posteriormente, de modo a conduzir uma reflexão sobre a complexidade de projetos de publicações digitais sistemáticas, realizou-se a construção de um conjunto de orientações, que se deu a partir da organização, sistematização e interpretação dos dados resultantes.

Como resultado, obteve-se uma visão inicial acerca das publicações digitais sistemáticas ${ }_{\llcorner}$decomposta em cinco eixos e dois subeixos, a saber: Conceitual, de Conteúdo (com os subeixos Organização e Fluxo), Funcional, de Experiência (com o subeixo Usabilidade) e Sensorial. Assim, o Quadro 1 caracteriza e exemplifica os eixos deste primeiro conjunto, o qual foi posteriormente submetido à consulta com especialistas.

\footnotetext{
${ }^{1}$ A revisão sistemática se dá por meio de buscas exaustivas em estudos publicados, apoiadas em um processo replicável, científico e transparente, que fornece um registro das decisões, procedimentos e conclusões dos pesquisadores.
} 
Quadro 1 - Primeira configuração dos eixos da publicação digital sistemática e exemplos de orientações.

\begin{tabular}{|c|c|c|}
\hline EIXO & DESCRIÇÃO & ORIENTAÇÕES \\
\hline Conceitual & $\begin{array}{l}\text { Envolve as definições iniciais da } \\
\text { publicação digital - as quais } \\
\text { fundamentalmente influenciarão nas } \\
\text { decisões dos demais eixos. Suas } \\
\text { orientações contemplam as } \\
\text { possibilidades potencializadas pelo } \\
\text { meio digital, bem como o objetivo e o } \\
\text { conceito do projeto. }\end{array}$ & $\begin{array}{l}7 \text { orientações. } \\
\text { Exemplo: } \\
\text { Considere os diferentes modos de } \\
\text { leitura, conforme o objetivo da } \\
\text { publicação ou o perfil do leitor: a } \\
\text { leitura linear, seletiva, consultiva, } \\
\text { informativa e suas implicações na } \\
\text { estrutura da publicação. }\end{array}$ \\
\hline $\begin{array}{l}\text { De } \\
\text { Conteúdo }\end{array}$ & $\begin{array}{l}\text { Diz respeito à definição e organização } \\
\text { do conteúdo, assim como à } \\
\text { estruturação da publicação, } \\
\text { possuindo dois subeixos: Organização } \\
\text { e Fluxo. }\end{array}$ & $\begin{array}{l}16 \text { orientações. } \\
\text { Exemplo: } \\
\text { Avalie a adequação do conteúdo ao } \\
\text { perfil ou objetivo da publicação. }\end{array}$ \\
\hline Funcional & $\begin{array}{l}\text { Agrupa as orientações que apontam } \\
\text { possíveis funcionalidades da } \\
\text { publicação digital e recursos } \\
\text { interativos, se referindo também ao } \\
\text { uso das diferentes mídias e } \\
\text { potencialidades do suporte. }\end{array}$ & $\begin{array}{l}11 \text { orientações. } \\
\text { Exemplo: } \\
\text { Considere o uso dos diferentes } \\
\text { recursos oferecidos pela plataforma ou } \\
\text { suporte (GPS, acelerômetro, câmera, } \\
\text { microfone, etc.), utilizando os cinco } \\
\text { sentidos na experiência. }\end{array}$ \\
\hline $\begin{array}{l}\text { De } \\
\text { Experiência }\end{array}$ & $\begin{array}{l}\text { Leva em consideração aspectos de } \\
\text { experiência do usuário, usabilidade e } \\
\text { ergonomia - possuindo o subeixo } \\
\text { Usabilidade. }\end{array}$ & $\begin{array}{l}13 \text { orientações. } \\
\text { Exemplo: } \\
\text { Considere uma navegação consistente, } \\
\text { fácil e clara, utilizando indicadores de } \\
\text { navegação e pistas de orientação, se } \\
\text { necessário. }\end{array}$ \\
\hline Sensorial & $\begin{array}{l}\text { Trata das dimensões de apresentação } \\
\text { e visualização do conteúdo. }\end{array}$ & $\begin{array}{l}10 \text { orientações. } \\
\text { Exemplo: } \\
\text { Atribua uma personalidade ao sistema } \\
\text { que vá ao encontro dos objetivos } \\
\text { criativos ou padrões de design. }\end{array}$ \\
\hline
\end{tabular}

Fonte: autores.

\section{PROCEDIMENTOS METODOLÓGICOS}

O processo de avaliação direcionado a especialistas - foco deste artigo -, teve o objetivo de verificar o conteúdo, a forma e a clareza das orientações desenvolvidas. Para tal, foi elaborado um questionário, realizado um teste piloto e posteriormente 
efetuadas as consultas finais. Finalmente, os resultados foram compilados e interpretados, realizando-se os ajustes e adaptações conforme as sugestões dos especialistas e, por conseguinte, a finalização do conjunto de orientações. Assim as principais etapas metodológicas foram:

- Elaboração do instrumento de pesquisa (questionário e documento de apresentação);

- Identificação do perfil dos especialistas a serem consultados;

- Aplicação do teste piloto;

- Aplicação dos questionários;

- Discussões.

\subsection{Instrumento de pesquisa}

O instrumento elaborado para realização da consulta com os especialistas foi composto por um questionário estruturado a partir de respostas pré-definidas e um documento de apresentação, apresentado na Figura 2. Tal instrumento teve como objetivo avaliar o conteúdo, a clareza e a forma das orientações desenvolvidas.

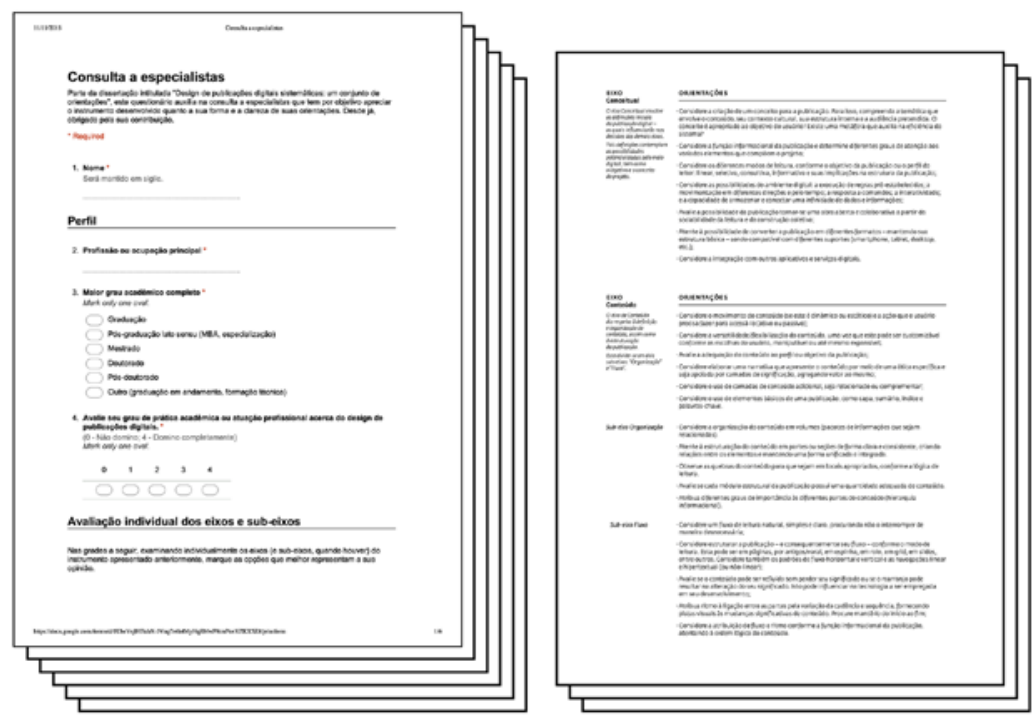

Figura 2 - Instrumento de consulta a especialistas.

Fonte: autores.

Assim, o documento de apresentação continha a descrição de cada eixo e subeixo, seguido de suas respectivas orientações. O questionário, por sua vez, era composto por três partes distintas: a primeira delas buscava compreender o perfil do especialista, e a segunda e terceira visavam avaliar o conteúdo, a clareza e a pertinência dos eixos, subeixos, orientações e do conjunto como um todo.

Desse modo, eram apresentadas asserções que diziam respeito à nomenclatura, clareza da descrição e adequação dos eixos e subeixos. Da mesma forma, para cada eixo e subeixo eram apresentadas afirmações a respeito da clareza e adequação das suas respectivas orientações. Ao especialista, portanto, cabia marcar seu grau de concordância com tais afirmações, em uma escala de cinco valores: discordo totalmente, discordo parcialmente, neutro, concordo parcialmente e 
concordo totalmente, havendo espaço para comentários qualitativos a respeito de cada eixo ou subeixo. Ao final, o mesmo tipo de avaliação era feita para o conjunto como um todo, a partir de asserções que diziam respeito ao número de eixos, à completude e à viabilidade de aplicação do conjunto.

\subsection{Especialistas consultados}

O grupo de especialistas foi composto por três profissionais e três professores ligados à área de publicações digitais. O especialista A é diagramador de publicações impressas e digitais em uma editora universitária e possui graduação em andamento. Já o especialista B é coordenador do laboratório de pesquisa da mesma editora universitária e também possui graduação em andamento. $O$ especialista $C$, por sua vez, intitula-se designer e instrutor em uma empresa de consultoria e treinamentos em publicações digitais e possui pós-graduação lato sensu.

O especialista $D$ é designer e professor doutor em uma instituição de ensino superior federal, na qual é responsável pelas disciplinas relacionadas à Tipografia e ao Design Editorial em um curso superior de Design. O quinto participante, especialista $E$, também é professor doutor em uma instituição de ensino superior federal, sendo responsável pela disciplina relacionada ao Design Editorial em um curso superior de Design. $O$ último consultado, especialista $F$, é professor doutor em uma instituição de ensino superior federal em um curso superior de Produção Editorial.

\subsection{Aplicação do teste piloto e aplicação dos questionários}

Após sua elaboração inicial, o instrumento de pesquisa foi previamente avaliado em um teste piloto realizado com um voluntário da área de Design Editorial, não havendo alterações em sua forma nem em seu conteúdo.

Em relação ao processo da consulta, inicialmente explanou-se sobre o conceito de publicação digital sistemática e apresentou-se o conjunto de orientações desenvolvido, por meio de um documento impresso. Após, com o documento em mãos, cada especialista respondeu de modo individual e presencial ao questionário aplicado, à exceção do especialista $C$, que teve sua consulta realizada por meio de um software de comunicação via internet, uma vez que este se encontrava na cidade do Rio de Janeiro, inviabilizando a consulta presencial. Dessa forma, o questionário foi realizado durante a segunda quinzena de Outubro de 2015.

\section{RESULTADOS E DISCUSSÕES}

Realizadas as consultas, os resultados foram compilados e são sintetizados a seguir. Iniciando-se pelo perfil dos participantes, o gráfico da Figura 3 apresenta o grau de prática acadêmica ou atuação profissional acerca do design de publicações digitais dos seis especialistas consultados. 


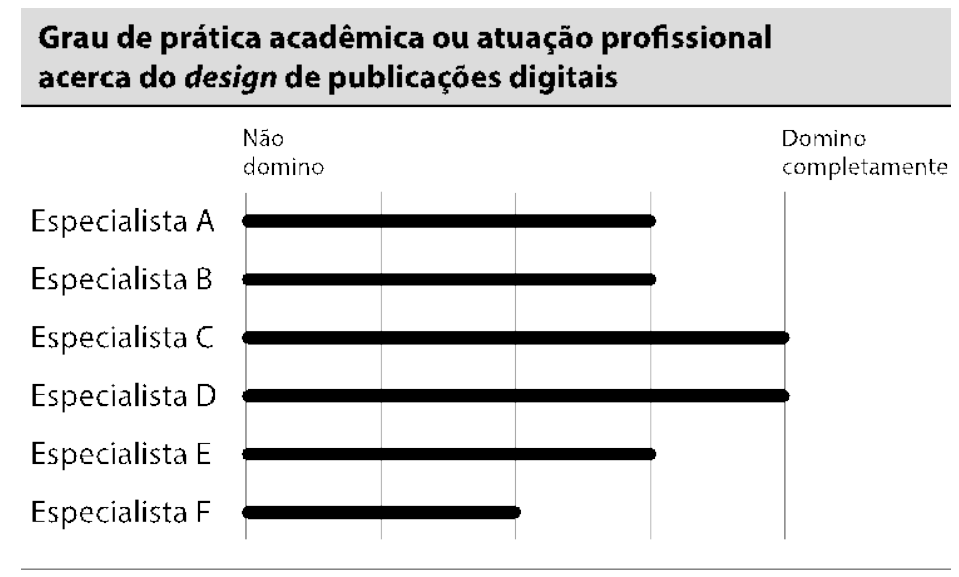

Figura 3 - Grau de prática acadêmica ou atuação profissional dos especialistas consultados. Fonte: autores.

Em relação à avaliação geral do conjunto de orientações, os especialistas concordam totalmente ou parcialmente que o número de eixos (cinco) está adequado. Apenas os especialistas B e $C$ sugeriram a criação de um novo eixo referente aos testes e validação e à distribuição. A mesma opinião se repetiu em relação à viabilidade da aplicação do instrumento na condução de projetos de publicações sistemáticas e no que se refere à completude do conjunto. Ou seja, os especialistas concordam que o instrumento tem sua aplicação viável e contempla a maioria dos aspectos deste tipo de projeto. Apenas o especialista $D$ afirmou que concorda parcialmente com a viabilidade de aplicação do conjunto - não explicitando as razões para sua opinião - e os especialistas $B$ e $C$ concordaram parcialmente que o conjunto contempla todos os aspectos do projeto de publicações digitais sistemáticas, uma vez que ambos sugeriram a criação de novos eixos.

Quatro participantes concordam que os nomes dos eixos e subeixos são adequados. Por outro lado, os especialistas $D$ e $F$ discordaram parcialmente do nome do eixo Sensorial, propondo a nomenclatura para eixo Visual ou Leiaute. Além disso, o especialista D se mostrou neutro em relação ao nome do eixo Funcional, sugerindo a renomeação para Funcionalidade.

Três dos seis especialistas concordaram totalmente que os todos os eixos e subeixos estão claros. O especialista A concordou parcialmente com a clareza do subeixo Fluxo, do subeixo Usabilidade, do eixo Sensorial e do eixo Conceitual. Em relação e este último, o especialista teve dúvidas relacionadas à diferença em entre publicações periódicas e sistemáticas. Apesar destes conceitos não serem abordados no eixo, foram explanados no início da pesquisa e, para o especialista, são importantes nas definições conceituais da publicação. Nos demais eixos ou subeixos, o especialista não deixou claro os motivos de sua escolha.

Ainda, o especialista C concordou parcialmente com a clareza do eixo de Conteúdo - sem explicitar o motivo no questionário. $O$ especialista $D$, por sua vez, concordou parcialmente com a clareza do eixo de Conteúdo e do eixo Funcional, pois acreditava que havia orientações do segundo que deveriam pertencer ao primeiro. Ainda, se mostrou neutro em relação à clareza do eixo Sensorial por discordar parcialmente da sua caracterização.

De maneira geral, os especialistas concordaram que as orientações contemplam o que os eixos e subeixos se propõem. Mais especificamente, o 
especialista A concordou parcialmente em relação ao subeixo Fluxo e ao subeixo Usabilidade; e o especialista $D$ concordou parcialmente em relação ao eixo de Conteúdo (pois esperava nele orientações relacionadas ao uso de mídias) e ao eixo de Experiência, onde esperava mais informações sobre motivação e percepção. Em relação ao eixo Sensorial, os especialistas A, D e $F$ afirmaram que concordavam parcialmente que suas orientações contemplavam o que o eixo se propunha, uma vez que os dois últimos não tiveram suas expectativas totalmente correspondidas. Nota-se que, novamente, o especialista $A$ não manifestou os motivos de suas escolhas.

De mesmo modo, a maioria dos especialistas concordou que as orientações dos eixos e subeixos estão claras. À exceção, o especialista A concordou parcialmente com a clareza das orientações do subeixo Organização, do subeixo Usabilidade e do eixo Sensorial sem manifestar o motivo. $O$ especialista $D$ concordou parcialmente em relação à clareza das orientações dos eixos Sensorial e de Conteúdo, pelo mesmo motivos citados anteriormente, como também em relação ao eixo Conceitual, sugerindo que uma das orientações fosse reescrita. O especialista $F$, por sua vez, concordou parcialmente em relação à clareza das orientações dos eixos Conceitual e Funcional, sem explicitar a razão de sua opinião.

Em relação à contribuição dos eixos, quatro dos seis especialistas concordaram que todos os eixos contribuem para o desenvolvimento de publicações digitais sistemáticas. À exceção, o especialista A concordou parcialmente em relação ao eixo Conceitual e o especialista $D$, ao eixo Sensorial, também não deixando claras as razões para tais opiniões.

No que diz respeito à adequação dos subeixos, cinco dos seis participantes concordaram que os subeixos são adequados aos seus respectivos eixos. À exceção, apenas o especialista $A$ concordou parcialmente em relação ao subeixo Fluxo e ao subeixo Usabilidade, não apresentando, porém, comentários ou contribuições nesse sentido.

Além disso, os especialistas também puderam contribuir por meio de comentários qualitativos no questionário - complementando sua avaliação objetiva. 0 Quadro 2 apresenta os principais comentários de cada especialista de acordo com o eixo ou subeixo avaliado.

Quadro 2 - Principais contribuições qualitativas advindas dos especialistas.

\begin{tabular}{|l|l|c|}
\hline DIMENSÃo & CONTRIBUIÇÃo & ESPECIALISTA \\
\hline $\begin{array}{l}\text { Eixo } \\
\text { Conceitual }\end{array}$ & $\begin{array}{l}\text { Elaborar melhor a orientação sobre obra aberta para torná- } \\
\text { la menos prescritiva. }\end{array}$ & D \\
\hline $\begin{array}{l}\text { Eixo de } \\
\text { Conteúdo }\end{array}$ & $\begin{array}{l}\text { Tratar da questão da natureza do conteúdo, ou seja, em } \\
\text { qual mídia será apresentado determinado conteúdo } \\
\text { (adequação). }\end{array}$ & D \\
\cline { 2 - 3 } & $\begin{array}{l}\text { Considerar outros elementos textuais na composição da } \\
\text { estrutura técnica de uma publicação (apresentação, folha } \\
\text { de rosto, etc.) }\end{array}$ & E \\
\hline $\begin{array}{l}\text { Subeixo } \\
\text { Organização }\end{array}$ & $\begin{array}{l}\text { Deixar mais claro o termo “partes do conteúdo". Sugestão: } \\
\text { elementos gráfico-editoriais (título, entretítulo, olho, linha } \\
\text { de apoio...) }\end{array}$ & E \\
\hline
\end{tabular}




\begin{tabular}{|c|c|c|}
\hline DIMENSÃO & CONTRIBUIÇÃO & ESPECIALISTA \\
\hline Subeixo Fluxo & $\begin{array}{l}\text { Conflito entre a orientação do eixo principal que trata da } \\
\text { adição de camadas de significação e a orientação "procure } \\
\text { não interromper o fluxo de maneira desnecessária". }\end{array}$ & A \\
\hline \multirow[t]{6}{*}{$\begin{array}{l}\text { Eixo } \\
\text { Funcional }\end{array}$} & $\begin{array}{l}\text { Considerar o controle de acesso à publicação e a proteção } \\
\text { contra cópia e compartilhamento. }\end{array}$ & $\mathrm{C}$ \\
\hline & $\begin{array}{l}\text { Avaliar o peso final da publicação em função do conteúdo } \\
\text { multimídia e interativo. }\end{array}$ & $\mathrm{C}$ \\
\hline & $\begin{array}{l}\text { Enfatizar a necessidade de relacionar as funcionalidades e o } \\
\text { uso de diferentes mídias de acordo com o } \\
\text { conceito/objetivo da publicação. }\end{array}$ & $\mathrm{F}$ \\
\hline & O uso de mídias poderia estar no eixo de Conteúdo. & D \\
\hline & Rever o nome do eixo. Sugestão: Funcionalidade. & D \\
\hline & Utilizar com cuidado as técnicas de notificação e alerta. & B \\
\hline \multirow[t]{2}{*}{$\begin{array}{l}\text { Eixo de } \\
\text { Experiência }\end{array}$} & $\begin{array}{l}\text { Sugerir de maneira mais direta a possibilidade de realizar } \\
\text { testes de navegabilidade/usabilidade no desenvolvimento } \\
\text { do projeto da publicação. }\end{array}$ & $\mathrm{F}$ \\
\hline & $\begin{array}{l}\text { Considerar uma maneira de enviar feedback a partir de } \\
\text { qualquer lugar da publicação (relatar um problema no } \\
\text { texto, por exemplo). }\end{array}$ & A \\
\hline Eixo Sensorial & Rever o nome do eixo. & $D ; F$ \\
\hline \multicolumn{3}{|c|}{ Comentários gerais } \\
\hline \multicolumn{2}{|c|}{ Incluir um eixo de teste/validação. } & B \\
\hline \multicolumn{2}{|c|}{$\begin{array}{l}\text { Vincular o instrumento a um texto de introdução/apresentação, } \\
\text { que explique de forma resumida seus objetivos e público prioritário. }\end{array}$} & $\mathrm{F}$ \\
\hline \multicolumn{2}{|c|}{ Incluir um eixo de distribuição. } & $\mathrm{C}$ \\
\hline
\end{tabular}

Fonte: autores.

Assim, após a compilação dos resultados, o conjunto de orientações foi modificado conforme as sugestões apresentadas anteriormente. Convém ressaltar que foram excluídos no ajuste final comentários cujos cunhos foram essencialmente técnicos, comentários de aspectos já tratados nas orientações, questões relativas a publicações periódicas (como periodicidade e assinatura), bem como definições mercadológicas (ligadas à venda e comercialização). Além disso, aprofundamentos a respeito de percepção e motivação foram descartados por estarem fora do escopo do estudo.

Nota-se, ainda, que foi necessária a adequação de algumas destas sugestões. Por possuir ênfase no artefato, a raiz do estudo não considerou uma visão mais ampla e sistêmica da publicação digital sistemática, assim questões de distribuição não foram contempladas. Além disso, algumas considerações foram realocadas de acordo com o 
seu grau de afinidade ao eixo. Ainda, comentários qualitativos realizados durante a consulta - mesmo que de maneira informal - também serviram de guia para algumas melhorias no conjunto.

Por fim, optou-se pela padronização na nomenclatura dos eixos, ocorrendo pequenas modificações em relação ao primeiro conjunto, bem como, a reordenação de algumas orientações.

\section{CONSIDERAÇÕES FINAIS}

Ao se avaliar o procedimento de consulta a especialistas, entende-se que sua realização fora muito importante para o fechamento da pesquisa. A visão particular acerca do design de publicações digitais de cada participante trouxe contribuições que não haviam sido identificadas na revisão de literatura ou que não tiveram sua importância devidamente atribuída.

Assim, visões dos especialistas acerca do resultado da pesquisa colaboraram para o seu refinamento, trazendo contribuições importantes que não constavam na primeira configuração do conjunto. Dentre estas, destaca-se a caracterização do eixo de Superfície - tendo em vista as orientações ali agrupadas e a contemplação dos aspectos sensoriais nos eixos de Experiência e de Funcionalidades -, a readequação deste último e do eixo de Conteúdo (no que tange o uso de mídias), além das questões de avaliação e testes da publicação durante o seu desenvolvimento e contribuições vinculadas à distribuição. Ainda, tal consulta possibilitou a verificação fundamental quanto à clareza, pertinência e adequação das orientações desenvolvidas.

Entretanto, o instrumento utilizado para a consulta - um questionário com respostas pré-definidas - apresentou algumas fragilidades que dificultaram a compilação dos resultados. A ausência da obrigatoriedade de justificativa para as opiniões diferentes de concordância total tornou algumas contribuições dos especialistas vagas e pouco pertinentes.

Mesmo assim, é importante destacar a realização da consulta a especialistas ao final da pesquisa, pois a partir das orientações estruturadas, os participantes puderam contribuir de maneira objetiva, uma vez que a elaboração das orientações requereu uma imersão teórica muito enraizada.

Para estudos futuros, aponta-se para a geração de outros possíveis eixos de projeto, como produção e distribuição, além da verificação do conjunto para a condução de projetos de outros tipos de publicações digitais, independentemente do estado da tecnologia vigente.

Finalmente, destaca-se que o conjunto de orientações resultante dos procedimentos aqui descritos - em sua forma final e organizado em cinco eixos: de Conceito, de Conteúdo (dividido em Organização e Fluxo), de Funcionalidades, de Experiência (com seu subeixo Usabilidade) e de Superfície - se encontra na integralidade disponível na dissertação de Dick (2015).

\section{REFERÊNCIAS}

AALTO (Finlândia). 24265 Course overview. 2014. Disponível em: <https://noppa.aalto.fi/noppa/kurssi/24265/esite>. Acesso em: 03 nov. 2014. 
COMPTON, Young Sun. Digital Publishing. In: LUPTON, Ellen (org.). Type on screen: A critical guide for designers, writers, developers \& students. Nova York: Princeton Architectural Press, 2014. p. 78-97.

COOPER, Alan. About Face 3: The Essentials of Interaction Design. Indianapolis: Wiley Publishing, 2007.

DICK, Maurício Elias; GONÇALVES, Berenice Santos. A análise de livro digital: uma visão de suas affordances. In: Anais do 110 Congresso Brasileiro de Pesquisa e

Desenvolvimento em Design. v. 1, n. 4. São Paulo: Blucher, 2014. p. 3275-3286. Disponível em:<http://www.proceedings.blucher.com.br/article-details/a-anlise-delivro-digital-uma-viso-de-suas-affordances-12907>. Acesso em: 04 maio 2015.

DICK, Maurício Elias. Design de publicações digitais sistemáticas: um conjunto de orientações. 2015. 175 f. Dissertação (Mestrado) - Programa de Pós-graduação em Design, Universidade Federal de Santa Catarina, Florianópolis, 2015.

GAVER, William W. Technology affordances. Cambridge, 2002. Disponível em: < http://www.cs.umd.edu/class/fall2002/cmsc434-0201/p79-gaver.pdf>. Acesso em: 10 maio 2014.

GIBSON, James J. The ecological approach to visual perception. New Jersey: LEA, 1986.

HASLAM, Andrew. O livro e o designer II: Como criar e produzir livros. São Paulo: Rosari, 2007.

HEIKKILÄ, Harri. Towards tablet publication heuristics. Aalto, Finlândia: 2013.

Disponível em: <http://www.vkl.fi/files/2525/Tutkimusraportti.pdf>. Acesso em: 20 jan. 2015.

LIDWELL, W; HOLDEN, K; BUTLER, J. Princípios Universais do Design. Porto Alegre: Bookman, 2010.

MOD, Craig. Books in the age of iPad. 2012. Disponível em:

<http://craigmod.com/journal/ipad_and_books/ >. Acesso em: 04 fev. 2015.

ROGERS, Y.; SHARP, H.; PREECE, J. Design de Interação: além da interação homemcomputador. Porto Alegre: Bookman, 2013.

SAMARA, Timothy. Guia de design editorial: Manual prático para o design de publicações. Porto Alegre: Bookman, 2011.

SCHERDIEN, Ingrid. O livro digital e as novas práticas de leitura: Proposição de Diretrizes Projetuais sob a Perspectiva do Design Estratégico. Dissertação de Mestrado. Universidade do Vale do Rio do Sinos. Porto Alegre, 2014. pp 171. Disponível em: <http://biblioteca.asav.org.br/vinculos/00000A/00000ABF.pdf>. Acesso em: 28 abr. 2015.

SCHLATTER, Tania; LEVINSON, Deborah. Visual Usability: Principles and Practices for Designing Digital Applications. Waltham: Morgan Kaufmann, 2013.

SILVA, Ana Catarina; BORGES, Maria Manuel. Book design program: a transition to a hybrid publishing context. Information Services \& Use, IOS Press, v. 31, p. 189-197, 2011. Disponível em: <http://ebooks.iospress.nl/publication/32008>. Acesso em: 03 nov. 2014. 